\title{
The clinicopathologic features and the validity of the new T1 pancreatic cancer defined by the 8th edition American Joint Committee on Cancer cancer staging system
}

\author{
Wooil KWON*
}

Department of Surgery, Seoul National University Hospital, Seoul, Korea

\begin{abstract}
Lecture: With the introduction of the eighth edition American Joint Committee on Cancer (AJCC) staging system for pancreatic cancer, several changes have been made to T1 pancreatic cancer. One of the most prominent changes was the removal of extrapancreatic extension concept, allowing many of the previously $\mathrm{T} 3$ tumors to be down staged to T1 pancreatic cancer. In addition, $\mathrm{T} 1$ was subcategorized into T1a, T1b, and Tlc discriminated by $0.5 \mathrm{~cm}$ and $1.0 \mathrm{~cm}$.

The down-staging of previous T3 tumors to T1 imply that surgeons and physicians will encounter more T1 pancreatic cancer patients than before. However, the prognosis and clinicopathologic features of T1 pancreatic cancer have not been investigated in depth in the past. In addition, because of the changes in the definition of T1 pancreatic cancer, the T1 pancreatic cancer cohort defined by the eighth edition AJCC system represents a new set of patients. With increasing T1 pancreatic cancer patients, there is a need for better understanding of clinicopathologic features and prognosis of T1 pancreatic cancers.

The feasibility of changes in T1 definition also needs to be investigated. The removal of the extrapancreatic extension concept was based on one study that did not present the relevant result in detail. Furthermore, prognostic insignificance of extrapancreatic concept was not reproduced in other studies. On the contrary, several studies have found extrapancreatic concept to have a prognostic significance.

Subcategorization was also a problematic change. The idea of subcategorization into Tla, T1b, and T1c was proposed by Dr. Adsay to categorize small invasive carcinomas associated with premalignant cystic lesions such as intraductal papillary mucinous neoplasm. This was a proposal, and the cut-off size values were not based on scientific evidence. Therefore, it is not clear why a proposal would be incorporated into a staging system.

To answer these questions, a study was initiated as a part of the 2nd Korea-Japan Collaborative Studies. Forty-two centers from Japan and Korea participated in this study and clinicopathologic data of 1,459 patients with T1 pancreatic cancer were collected for analysis. The patients were resected between 2000 and 2019. Subtypes other than pancreatic ductal adenocarcinoma and pancreatic cancers arising from premalignant cystic lesions were excluded. Patients who did not have lymph nodes assessed or had distant metastasis at surgery were excluded. Patients who received neoadjuvant treatment were also excluded from the study.

This lecture aims to deliver the clinicopathologic features and the outcome of T1 pancreatic cancer. At the same time, this lecture will investigate the feasibility of the T1 definition by the eighth edition AJCC staging system for pancreatic cancer and demonstrate a need for revision in the future.
\end{abstract}

